\title{
Criação de um jogo para desenvolver o Pensamento Computacional percorrendo caminhos eulerianos
}

\author{
Luiz Alencar $^{1}$, Fernanda Pires $^{1}$, Marcela Pessoa ${ }^{1}$ \\ ${ }^{1}$ Universidade do Estado do Amazonas (UEA) - Escola Superior de Tecnologia (EST) - \\ Laboratório de Pesquisa e Desenvolvimento em Tecnologias Educacionais (ThinkTEd) \\ Manaus - AM - Brasil \\ \{lfba.lic17, fpires, msppessoa\}@uea.edu.br
}

\begin{abstract}
This paper presents a proposal for an educational game called " $O$ seqüestro de Magrafo", whose objective is to assist in the learning of Eulerian paths in the graph, in addition to the exercise or development of Computational Thinking skills. The educational evaluation model of MEEGA + KIDS games with elementary school children was applied, which evidences the game can be a promising tool for auxiliary learning..
\end{abstract}

Resumo. Este artigo apresenta uma proposta de jogo educacional, denominado "O sequestro de Magrafo", cujo objetivo é auxiliar na aprendizagem de caminhos eulerianos em grafo e exercitar o desenvolvimento das habilidades de Pensamento Computacional. Foi aplicado o modelo de avaliação educacional de jogos MEEGA+KIDS, com crianças do Ensino Fundamental, que dá indícios de que o jogo pode ser uma ferramenta promissora para auxiliar na aprendizagem.

\section{Introdução}

O Pensamento Computacional (PC) é defendido como um conjunto de habilidades que permite a resolução de problemas de forma otimizada, cujo desenvolvimento é tão importante quanto Língua Portuguesa e Matemática, por isso deveria ser explorado na escola [Wing 2006]. O conceito é amplamente abordado em Ciência da Computação que, de forma recorrente, faz uso dos seus quatro pilares: decomposição, reconhecimento de padrões, abstração e algoritmos.

O desenvolvimento do Pensamento Computacional parece estar diretamente ligado à capacidade de visualizar e interpretar problemas para então agir sobre eles, tal como ocorre a aprendizagem, através do reconhecimento de erros que emitem feedback [Piaget and Inhelder 2008]. Pesquisas apontam os jogos educacionais como estratégias lúdicas para exercitar as habilidades de resolução de problemas, bem como desenvolver níveis de abstração através da construção de modelos mentais [Michel et al. 2019, Pessoa et al. 2019]. Neste sentido, este artigo descreve o desenvolvimento do jogo educacional intitulado "O Sequestro de Magrafo", por estudantes de Licenciatura em Computação, cujo objetivo é promover o desenvolvimento do Pensamento Computacional, através de grafos eulerianos. São apresentados problemas de caminhos eulerianos, em que o jogador deve traçar uma estratégia para passar por todas as rotas uma única vez. O jogo contempla aspectos da teoria cognitiva de aprendizagem construcionista [Piaget and Inhelder 2008], uma vez que o usuário ao progredir no jogo desenvolve habilidades de tomada de decisões. Assim, por meio de tentativas, é possível elaborar novas estratégias, sendo o erro um dos principais fatores para aprendizagem. 
O restante deste artigo está organizado como segue: na Seção 2 são apresentados alguns trabalhos relacionados e a fundamentação teórica, na Seção 3 é descrita a proposta do jogo, na Seção 4, os resultados preliminares e na Seção 5 as considerações finais.

\section{Grafos e a representação de problemas computacionais em jogos}

Grafos são estruturas matemáticas robustas utilizados para a representação de problemas. Um grafo $G=(V, E)$ pode ser definido como um um conjunto não vazio de vértices $(V)$ e um conjunto de arestas (E). Cada vértice pode ter uma ou mais arestas associadas a ele.

Em 1763, o matemático Leonard Euler formalizou um clássico problema de grafos a partir do seguinte cenário: no Rio Pregel, na cidade de Konigsberg, existem duas ilhas formando quatro regiões, com sete pontes interligando-as. O problema consiste em, partindo de uma dessas regiões, determinar um trajeto pelas pontes segundo o qual se possa retornar à região de partida, após atravessar cada ponte exatamente uma vez. Entretanto, Euler mostrou que não existe tal trajeto e utilizou um modelo em grafos para uma generalização desse problema. Através desse modelo ele verificou que circuitos como o descrito só existem quando e somente quando cada região tiver um número par de pontes [Szwarcfiter 2018].

O jogo proposto utiliza o conceito de caminho euleriano (uma rota em um grafo que se visita todas as arestas exatamente uma vez). Para ajudar na fixação do conceito no jogo, todas as vezes que o usuário percorre uma aresta, esta é eliminada, não permitindo que seja reutilizada, desta forma, é garantida a propriedade de caminho euleriano.

Assim como há dificuldade no entendimento dos conceitos relacionados a grafo, há também dificuldade no entendimento de outros problemas clássicos da Computação, o que tem despertado o interesse de pesquisadores em propor soluções que envolvam ludicidade. Entre eles, Michel et al. (2019) apresentam o "WAlgor", um jogo que permite utilizar conceitos de problemas como busca, ordenação, coloração de grafos e o problema da mochila para avançar de fase. O jogo apresenta a temática "tower defense" pois é preciso posicionar o cavaleiro para se proteger e o jogador aplica algoritmos clássicos para vencer. Existem também jogos que se preocupam com a aprendizagem de lógica de programação para crianças, como é o caso de "Looking for Pets" [Pessoa et al. 2019] e "A Máquina do Curupira" [Pires et al. 2019], que exploram os quatro pilares do Pensamento Computacional através da criação de algoritmos.

O jogo proposto, "O Sequestro de Magrafo", foi projetado para auxiliar no entendimento de conceitos de caminhos em grafos através de elementos visuais, assim como "WAlgor". Semelhante a "Looking for Pets" e "A Máquina do Curupira" o jogador precisa elaborar um algoritmo para percorrer os grafos e progredir no jogo, com o diferencial de ter que traçar rotas.

\section{Descrição do jogo e processo de desenvolvimento}

"O Sequestro de Magrafo" é um jogo educacional para aprendizagem de grafos em que o jogador desenvolve as habilidades do Pensamento Computacional ao mesmo tempo em que exercita conceitos de grafos. O jogo também pode ser utilizado em diferentes

\footnotetext{
${ }^{1}$ Disponível em: github.com/LuizAlencar17/o-sequestro-de-magrafo
} 
contextos, trabalhando noções iniciais de algoritmos, bem como conceitos mais abstratos de grafos, de forma lúdica. Os elementos do jogo como pontos, caixas coloridas e caminhos dinâmicos podem gerar sensações de desafio e entusiamo nos usuários. Neste sentido o jogo pode ser utilizado como uma ferramenta de aprendizagem para estudantes de graduação, adolescentes e crianças.

Cada fase do jogo possui um grafo formado por vértices coloridos. O jogador deve direcionar o personagem principal através de um algoritmo de cores, ou seja, uma sequência de vértices para caminhar, contudo ao passar por uma aresta o caminho de volta ficará inacessível. A Figura 1 apresenta uma sequência de telas do jogo, iniciando pela tela de apresentação, seguida de algumas fases do jogo. Os vértices do gráfico estão em quadrados coloridos, conectados pelas as arestas. A Figura 2 apresenta a evolução do conceito de grafos e como os elementos de grafos são encontradas no jogo.

O jogo também foi elaborado para exercitar os quatro pilares do Pensamento Computacional através da gameplay e elementos do jogo. A Tabela 1 identifica as habilidades do Pensamento Computacional presentes no jogo.
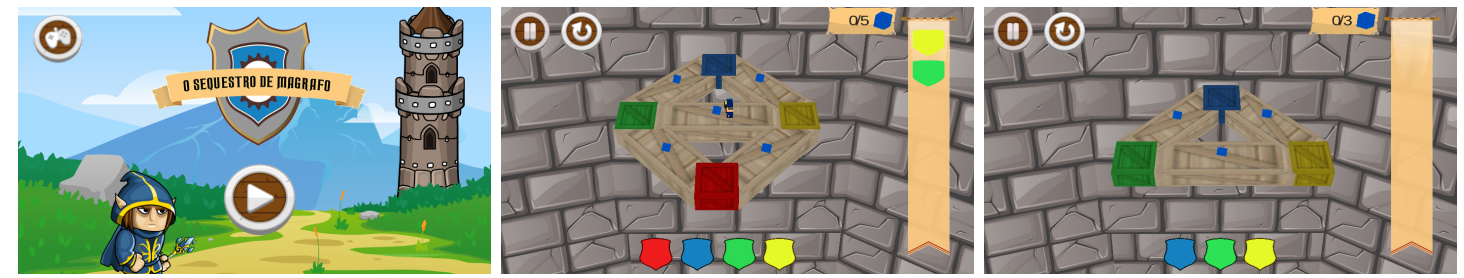

Figura 1. Sequência de telas do Jogo "O Sequestro de Magrafo".

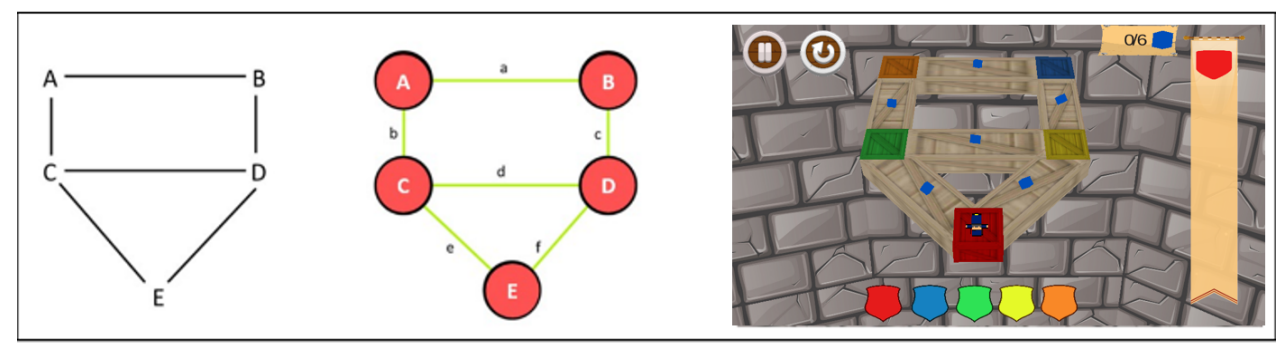

Figura 2. Características de grafos no jogo.

O jogo foi desenvolvido na linguagem C\#, na plataforma Unity. Foi usado um processo de desenvolvimento iterativo e incremental, utilizado em outros trabalhos [de Sousa Pires et al. 2020, Michel et al. 2019]. O processo é dividido nas seguintes etapas: i) Idealização: pesquisa e investigação do problema de aprendizagem; ii) Elicitação e análise de requisitos: levantamento e verificação de requisitos de aprendizagem, mecânica e elementos; iii) Planejamento: organização de atividades a serem realizadas; iv) Protótipo de baixa fidelidade: protótipo visual feito em papel; v) Protótipo de média fidelidade: primeira versão do protótipo para celular; vi) Protótipo de alta fidelidade: última versão do protótipo com bugs e problemas removidos; vii) Validação de protótipos: todos os protótipos foram verificados afim de validar elementos e proposta; viii) Aplicação de testes finais: aplicação de teste heurístico com estudantes do Ensino Médio; e ix) Documentação: o Game Document Design (GDD), documento que descreve detalhes do jogo. 
Tabela 1. Pensamento Computacional e "O Sequestro de Magrafo"

\begin{tabular}{|l|l|l|}
\hline $\begin{array}{l}\text { Pensamento } \\
\text { Computacional }\end{array}$ & \multicolumn{1}{|c|}{ Descrição } & \multicolumn{1}{c|}{ O Sequestro de Magrafo } \\
\hline Decomposição & $\begin{array}{l}\text { Processo de divisão de um } \\
\text { problema grande e com- } \\
\text { plexo em problemas meno- } \\
\text { res e de baixa complexidade }\end{array}$ & $\begin{array}{l}\text { Os elementos do jogo são divididos em } \\
\text { elementos da interface (botões) e ele- } \\
\text { mentos de gameplay (personagem prin- } \\
\text { cipal, estrutura da fase) }\end{array}$ \\
\hline $\begin{array}{l}\text { Reconhecimento } \\
\text { de padrão }\end{array}$ & $\begin{array}{l}\text { Identificação de carac- } \\
\text { terísticas e propriedades } \\
\text { semelhantes }\end{array}$ & $\begin{array}{l}\text { As fases seguem um padrão, o jogador } \\
\text { precisa sempre encontrar um caminho } \\
\text { euleriano em um grafo para progredir }\end{array}$ \\
\hline Abstração & $\begin{array}{l}\text { Definição das partes e ele- } \\
\text { mentos mais importantes de } \\
\text { um problema }\end{array}$ & $\begin{array}{l}\text { As partes mais importantes do jogo são } \\
\text { os vértices coloridos que o jogador deve } \\
\text { caminhar }\end{array}$ \\
\hline Algoritmo & $\begin{array}{l}\text { Sequência de passos lógica } \\
\text { para uma possível solução }\end{array}$ & $\begin{array}{l}\text { Para caminhar o jogador dever criar um } \\
\text { algoritmo de cores indicando a sequência } \\
\text { de vértices a ser visitada }\end{array}$ \\
\hline
\end{tabular}

\section{Resultados preliminares}

O jogo está em fase de testes para verificar sua viabilidade quanto a ferramenta e aprendizagem, para isso, passou por uma avaliação heurística seguindo o modelo de avaliação de jogos educacionais chamado MEEGA+KIDS [Von Wangenheim et al. 2018], com questões reduzidas. O teste aplicado analisou dez métricas: Estética, Aprendizibilidade, Operabilidade, Confiança, Desafio, Satisfação, Diversão, Atenção focada, Relevância e Percepção da aprendizagem, como apresentado no Gráfico 3.

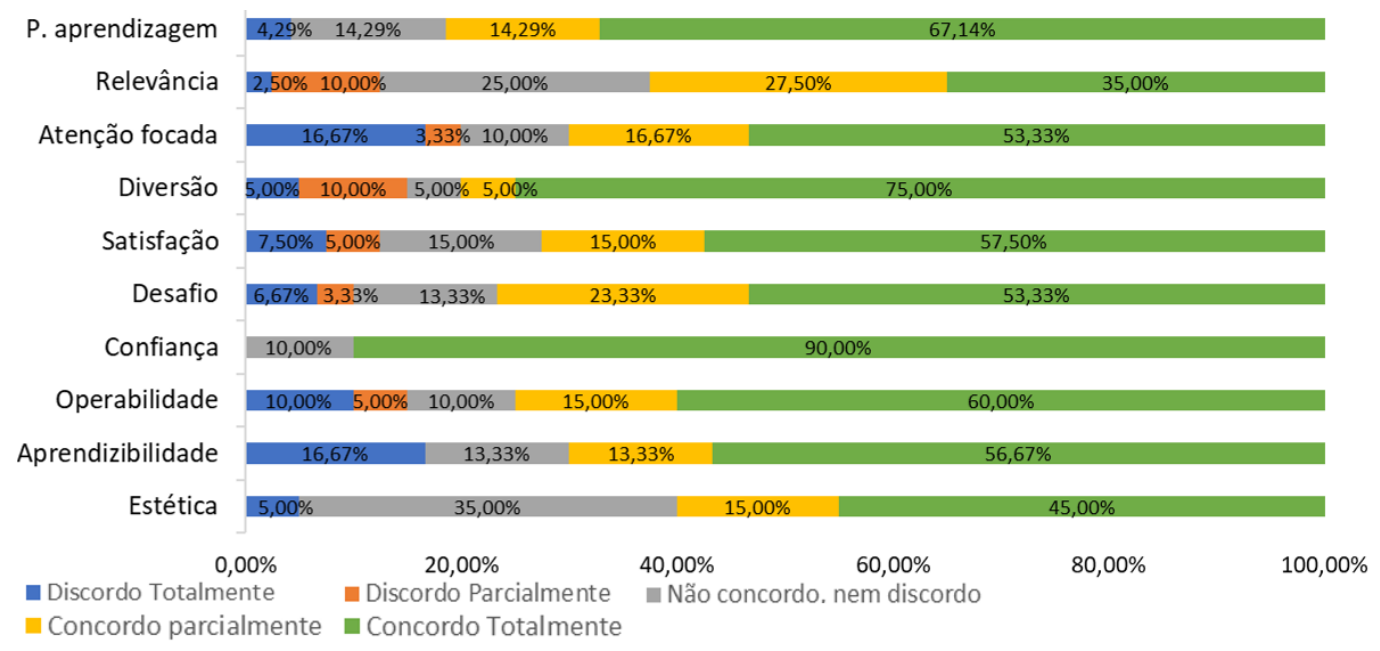

Figura 3. Avaliação do jogo com o Meega+Kids.

A avaliação foi realizada por treze estudantes do Ensino Fundamental do nono ano, com idades entre dez e treze anos. Cada métrica é formada por um conjunto de perguntas, sendo pontuado usando a escala likert de 05 pontos com pesos de -2 a 2 .

É possível observar que a métrica "Confiança" tem o melhor desempenho, com $90 \%$ de respostas entre concordo e concordo fortemente. Já a métrica "Estética", apresentou o pior desempenho com $60 \%$ entre concordo e concordo fortemente, o que era esperado considerando que o jogo ainda precisa de ajustes de design visual, porém, pare- 
ceu não interferir nos desafios apresentados e na confiança, mas, tudo indica que interferiu na atenção focada. Isso fornece indícios de que os requisitos estéticos tem alto grau de importância na confecção de um jogo para aprendizagem.

\section{Considerações Finais}

Este artigo apresenta o jogo "O Sequestro de Magrafo", um jogo educacional para auxiliar na aprendizagem de conceitos de caminhos em grafos e a desenvolver o Pensamento Computacional. O processo de desenvolvimento envolveu a elaboração de protótipos, validação e teste heurístico com crianças do Ensino Fundamental.

A avaliação do jogo apresenta resultados positivos (nota 1 e 2) quanto às métricas de Confiança, com 90,00\%, Percepção da aprendizagem com $81,43 \%$ e Diversão com $80,00 \%$. Os resultados indicam que o jogo é uma possível ferramenta para engajar estudantes a exercitar conceitos de caminhos em grafos.

Para trabalhos futuros pretende-se realizar algoritmos no jogo com intuito de capturar dados e logs dos jogadores, para, então, identificar a evolução de sua aprendizagem no jogo.

\section{Referências}

de Sousa Pires, F. G., Pessoa, M. S. P., Ferreira, R. M., Bernardo, J. R. S., and de Lima, F. M. M. (2020). O livro do conhecimento: um serious game educacional para aprendizagem de ortografia da língua portuguesa. Revista Brasileira de Informática na Educação, 28:436.

Michel, F., Pires, F., and Pessoa, M. (2019). Walgor: um jogo de tower defense para o desenvolvimento do pensamento computacional e apresentação de algoritmos computacionais. In Anais dos Workshops do Congresso Brasileiro de Informática na Educação, volume 8 , page 514 .

Pessoa, M., Alencar, L. F., Araújo, L., Melo, R., and Pires, F. (2019). Looking for pets: a game for the logical reasoning development. In 2019 IEEE Frontiers in Education Conference (FIE), pages 1-4. IEEE.

Piaget, J. and Inhelder, B. (2008). The psychology of the child. Basic books.

Pires, F., Teixeira, K., Pessoa, M., and Lima, P. (2019). Desenvolvendo o pensamento computacional através da máquina de Turing: o enigma do curupira. In Anais do XXVII Workshop sobre Educação em Computação, pages 523-532. SBC.

Szwarcfiter, J. (2018). Teoria computacional de grafos: Os Algoritmos. ELSEVIER, Rio de Janeiro.

Von Wangenheim, C. G., Petri, G., and Borgatto, A. F. (2018). Meega+ kids: A model for the evalua-tion of educational games for compu-ting education in secondary school. INCoD - Brazilian Institute for Digital Convergence.

Wing, J. M. (2006). Computational thinking. Communications of the ACM, 49(3):33-35. 